Themenheft Nr. 45:

Pädagogisches Wissen im Lichte digitaler und datengestützter Selbstoptimierung Herausgegeben von Estella Ferraro, Sabrina Schröder und Christiane Thompson

\title{
Etablierung normativer Ordnungen als Spielarten optimierter Selbstführung?
}

\section{Die Regierung des Pädagogischen am Beispiel des $4 \mathrm{~K}$ - und $21^{\text {st }}$-Century- Skills-Diskurses}

Patrick Bettinger ${ }^{1}$ (D)

${ }^{1}$ Pädagogische Hochschule Zürich

\begin{abstract}
Zusammenfassung
Seit einigen Jahren sind unter den Schlagworten der $\left\langle 21^{\text {st }}\right.$ Century-Skills $\rangle$ bzw. der $44 \mathrm{~K}$ Skills insbesondere im englischsprachigen Raum Bemühungen erkennbar, das Bildungssystem umfassend zu reformieren. Dabei spielt besonders die digitale Transformation eine entscheidende Rolle, da diese einerseits als ein Grund für den Reformbedarf des Bildungssystems vorgebracht wird und andererseits massgeblich die inhaltliche Zielrichtung der angestrebten Veränderungen bestimmt. Bei genauerer Betrachtung fällt auf, dass sich hinter diesem Reformdiskurs ein vielschichtiges globales Netzwerk unterschiedlicher Akteurinnen und Akteure aus dem Bildungsbereich, der Politik und insbesondere auch aus der Wirtschaft verbirgt. Der Beitrag beleuchtet diese Zusammenhänge aus einer gouvernementalitätstheoretischen Blickrichtung und fragt nach den hier erkennbaren Formen von Selbst- und Fremdführung. Dabei wird besonderes Augenmerk auf die so hervortretenden Techniken der Normalisierung gelegt, die sowohl einer Disziplinarlogik folgen als auch deutliche Merkmale von auf Prävention ausgelegten Sicherheitstechnologien aufweisen.
\end{abstract}

Establishing Normative Orders as Varieties of Optimized Self-Direction? The Government of the Pedagogical on the Example of the $4 \mathrm{~K}$ and $21^{\text {st }}$ Century Skills Discourse

\begin{abstract}
For some years now, efforts to comprehensively reform the education system have been discernible under the catchwords of (21st Century Skills» or 44 C Skills», especially in the English-speaking world. In this context, the digital transformation plays a particularly decisive role, as it is cited on the one hand as a reason for the need to reform the education system and, on the other hand, as a significant factor in determining the direction of the
\end{abstract}


content of this desired change. A closer look reveals that behind this reform discourse lies a complex global network of different actors from the education sector, politics and, in particular, economics. This article examines these interrelationships from a governmentality-theoretical perspective and questions the forms of leadership that can be discerned here. Special attention is paid to the techniques of normalization that emerge in this way, which follow a disciplinary logic and exhibit clear characteristics of security technologies designed for prevention.

\section{Einleitung und Problemabriss}

Der insbesondere im US-amerikanischen Kontext präsente Ansatz des 4K-Modells des Lernens (bzw. im Original 4C-Skills) erhält seit einigen Jahren auch im deutschsprachigen Raum vermehrt Aufmerksamkeit. Unter den Begriffen 〈Kreativität), ‘kritisches Denken`, 〈Kollaboration` und ‘Kommunikation` werden zentrale Kompetenzen gefasst und als übergreifende Zielsetzung für Lernprozesse in unserer Gegenwartsgesellschaft verstanden. Urheber des Konzepts ist das Netzwerk Partnership for 21st Century Skills, das als Verbund unterschiedlichster Wirtschaftsunternehmen sowie Non-Profit- und Bildungsorganisationen bereits vor mehr als zehn Jahren in Form verschiedener Publikationen (z. B. Rotherham und Willingham 2009; Bellanca und Brandt 2010) eine Kampagne zur Reform des Bildungswesens anstiess. Diese Kampagne strebte die Setzung bestimmter Lernziele - subsumiert als 4K-Skills - an, die als notwendige Reaktion des Bildungswesens auf eine veränderte (Arbeits-)Welt verstanden werden. Erklärtes Ziel der Organisation ist

«[...] to serve as a catalyst for $21^{\text {st }}$ century learning by building collaborative partnerships among education, business, community, and government leaders so that all learners acquire the knowledge and skills they need to thrive in a world where change is constant and learning never stops». ${ }^{1}$

Es lässt sich beobachten, dass in den vergangenen Jahren verschiedene Initiativen und Organisationen gegründet wurden, die sich für eine Implementation des 4K-Modells im Bildungssystem stark machen (bspw. das Center for Curriculum Rede$\left.s i g n^{2}\right)$. Umgesetzt wird dies unter anderem durch entsprechende Ratgeberliteratur sowie Beratungs- und Weiterbildungsangebote für Akteurinnen und Akteure aus dem Bildungsbereich. Zudem sprechen sich wirtschaftliche und politische Organisationen wie etwa die OECD sehr offensiv für eine Orientierung des Bildungssystems an den 4 K-Skills aus. ${ }^{3}$

1 https://www.educationinnovations.org/p/partnership-for-21st-century-skills-p21.

2 https://curriculumredesign.org.

3 https://www.oecd.org/general/thecasefor21st-centurylearning.htm. 
Die 4K-Skills reklamieren explizit den Anspruch der Zielbestimmung von Lernund Bildungsprozessen, deren Legitimierung zum Teil sehr deutlich an wirtschaftlicher Verwertbarkeit festgemacht wird. So hält Stauffer (2020) prägnant fest: «These skills are intended to help students keep up with the lightning-pace of today's modern markets». Schlagworte zur Begründung dieser Setzung sind darüber hinaus 〈Nachhaltigkeit) und ‘Gemeinwohl〉 als Zielsetzung von Bildung, die im Sinne einer umfassenden Persönlichkeitsformung neben den genannten Kompetenzen auch die Prägung von bestimmten «Charaktereigenschaften, die erfüllten Menschen helfen, mit anderen zusammenzuleben, zusammenzuarbeiten und eine nachhaltige Menschheit aufzubauen» (Fadel, Bialik, und Trilling 2017, 2), mit sich bringen sollen. Auch an anderer Stelle finden sich Verweise, die sich für eine Implementierung des 4K-Modells im Bildungssystem aussprechen und ihre Argumentation an klassischen pädagogischen Begründungslinien, wie der Befähigung zur gesellschaftlichen Teilhabe orientieren: «[...] 4K sind die Kompetenzen, die ein Mensch im 21. Jahrhundert braucht, um einerseits in der Gesellschaft zu bestehen und andererseits diese mitzugestalten» (Sterel, Pfiffner, und Caduff 2018).

Es lassen sich in den letzten Jahren bildungspolitische Bemühungen erkennen,

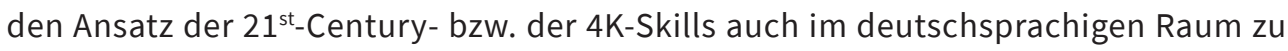
stärken und entsprechende Anpassungen in den Bildungssystemen vorzunehmen. Exemplarisch zeigt sich dies etwa anhand von Aktivitäten des Hochschulforums Digitalisierung, respektive dem Stifterverband für die Deutsche Wissenschaft. ${ }^{4}$ Erste curriculare Verankerungen des Ansatzes liegen z. B. auch in Form von Studiengangsreformen ${ }^{5}$ oder als Fort- und Weiterbildungsangebote an Fachhochschulen ${ }^{6}$ und Universitäten ${ }^{7}$ vor. Zudem greifen zahlreiche Multiplikatorinnen und Multiplikatoren im Schnittfeld von Bildungspolitik und pädagogischer Praxis den Ansatz auf (siehe auch die in diesem Zusammenhang gegründete Zentralstelle für Lernen und Lehren im 21. Jahrhundert ${ }^{9}$ ). Aufwind bekommt der Diskurs im deutschsprachigen Raum seit einiger Zeit insbesondere durch die Frage nach einer angemessenen Reaktion des Bildungssystems angesichts der fortschreitenden Digitalisierung (Aufenanger 2017; Kreß 2018).

4 https://hochschulforumdigitalisierung.de/de/blog/kompetenzen-21st-century-skills bzw. https://hochschulforumdigitalisierung.de/de/news/future-skills-nationales-aktionsprogramm-fuer-bildung-im-digitalen-zeitalter oder https://hochschulforumdigitalisierung.de/sites/default/files/dateien/Diskussionspapier3_Haltung_als_wichtiger_Bestandteil.pdf.

5 https://phzh.ch/globalassets/phzh.ch/ausbildung/sek2/semesterplan_abu_4k-modell_hs21.pdf.

6 https://www.hs-rm.de/de/service/didaktik-und-digitale-lehre/future-skills-welche-anforderungen-stelltdie-digitalisierung-an-die-studierenden.

7 https://www.uni-bonn.de/einrichtungen/pe-karriere/21st-century-skills.

8 https://www.joeran.de/die-4k-skills-was-meint-kreativitaet-kritisches-denken-kollaboration-kommunikation/.

9 https://zll21.de. 
Der Beitrag setzt an diesen Beobachtungen an und befasst sich aus gouvernementalitätsanalytischer Perspektive mit dem Diskurs um 4K-/21 $1^{\text {st }}$-Century-Skills. In diesem Zusammenhang wird untersucht, welche diskursiven Strategien der Etablierung normativer Ordnungen sich im Zusammenhang mit $4 \mathrm{~K}-/ 21^{\text {st-Century-Skills }}$ zeigen und inwiefern hierbei Formen der Selbst- und Fremdführung von Bedeutung sind. Vor dem Hintergrund der gouvernementalitätstheoretischen Grundlegung, die davon ausgeht, «dass Praktiken der Subjektivierung mit der fortschreitenden Moderne Individuen immer weniger über klassische Disziplinierung objektivieren, sondern dass diese dazu gebracht werden, Praktiken der Subjektivierung auf sich selbst anzuwenden und sich so als Subjekte zu subjektivieren» (Fegter et al. 2015, 25), beleuchtet der Beitrag ebensolche Verschränkungen von Formen der Selbst- und Fremdregierung, die im Rahmen des genannten Phänomenbereichs in Erscheinung treten. Damit geraten die «institutionellen Arrangements und Expertensysteme, Ordnungskategorien und Sortierverfahren, Lernprogramme und Sanktionsmechanismen, (Selbst-)Beobachtungs- und (Selbst-)Modellierungsprozeduren» (Bröckling 2007, 25) als konstitutive Ausgangspunkte von Formen der Subjektivierung in den Blick. Diesbezüglich wird zunächst gefragt, welche Konstellationen das diskursive Feld der $4 \mathrm{~K}-/ 21^{\text {st }}$-Century-Skills hervorbringt. Somit wird das Bedingungsgefüge beleuchtet, von dem ausgehend jene Macht-Wissen-Komplexe generiert werden, die dieses Feld strukturieren. Gerade der Rekurs auf den Begriff der «Kreativität) im Rahmen des 4KModells sowie die ökonomischen Verstrickungen legen nahe, die konzeptionelle Folie des ‘unternehmerischen Selbst - als eine Erscheinungsform neoliberaler Gouvernementalität - heranzuziehen, um das Verhältnis und die Bruchlinien zwischen Formen von Subjektivierungs- und Optimierungsfiguren zu betrachten. Durch die dieser Herangehensweise inhärente Unterscheidung von Herrschafts- und Selbsttechniken (Maurer und Weber 2006, 10) eröffnen sich Analyseoptionen, die insbesondere für die Frage sensibilisieren, wie sowohl Normen (verstanden als präskriptive, wertebasierte und sozio-kulturell figurierte Handlungsregulation) als auch Normalisierungen (verstanden als Konstruktion von Gewöhnlichkeit, Durchschnitt und Selbstverständlichkeit) auf unterschiedlichen Ebenen innerhalb dieses diskursiven Gefüges (re-)produziert werden (Schröder und Wrana 2015, 11ff.).

Der Beitrag schliesst hier an Überlegungen zu Formen und Modi von Normalisierung im Feld des Pädagogischen an (Bühler et al. 2015), indem er «nach dem Verhältnis von Normen und Normalität zu Subjektivität [fragt] und danach, wie sich Subjektivierung auch über Normalisierung vollzieht» (Schröder und Wrana 2015, 11). Den Ausgangspunkt dieser Analyse bildet eine Auseinandersetzung mit ausgewählten Dokumenten (Büchern, Fachbeiträgen, Frameworks, Webseiten, Dokumentationen) des $4 \mathrm{~K}-/ 21^{\text {st }}$-Century-Skills-Diskurses. Diese werden im Sinne von Diskursfragmenten gelesen, welche das Feld möglicher Positionen strukturieren, indem sie Ein- und Ausschliessungen vornehmen und somit eine regulatorische Funktion erfüllen, die 
spezifische Sag- und Sichtbarkeiten legitimiert. Hierzu wird nach übergreifenden und sich wiederholenden Mustern im Sinne diskursiver Äusserungsakte gesucht, um so die gouvernementale «Formierung der Normalisierungsmacht» (ebd., 18) zu identifizieren und aus pädagogischer Sicht zu diskutieren. Dabei wird besonderes Augenmerk auf die Frage gelegt, inwiefern hier Figuren optimierter Selbstführung relevant werden. Diese Schwerpunktsetzung ist - so die hier vertretene These - aus (medien-) pädagogischer Sicht deshalb von besonderer Relevanz und anschlussfähig, weil sich bereits an anderer Stelle im Hinblick auf mediale Verschränkungen des Pädagogischen Formen von (Selbst-)Kontrolle gezeigt haben, wie etwa im Rahmen der Praxis des Führens von E-Portfolios (Münte-Goussar 2011). Die hier identifizierte Kopplung von gouvernementaler Kontrolltechnik und einer spezifischen Form der Selbstsorge lässt sich als Optimierung im Zeichen neoliberaler Steuerungslogiken verstehen. Diese Optimierung ist durch eine Ambivalenz gekennzeichnet: Einerseits aktualisiert sie durch «Selbst-Kontrolle, Selbst-Ökonomisierung und Selbst-Rationalisierung» (ebd., 236) die charakteristische Logik gegenwärtiger Arbeitsformen des unternehmerischen Selbst (Bröckling 2007); andererseits verspricht sie gerade darüber Möglichkeiten der Autonomie und Selbstbestimmung.

\section{2. (Gouvernemetalität) als analytische Rahmung in der (Medien-)Pädagogik}

Die Auseinandersetzung mit Fragen von Macht, Wissen und Steuerung stellt seit Langem einen zentralen Interessensbereich der Erziehungswissenschaft und ihrer unterschiedlichen Felder dar. Gleichwohl zeigen sich deutliche Unterschiede in Bezug auf die Intensität der Auseinandersetzung mit Foucaults Gouvernementalitätsansatz mit Blick auf verschiedene subdisziplinäre Bereiche. Während unter anderem in der allgemeinen Erziehungswissenschaft ein reger Diskurs um den Ansatz beobachtet werden kann, zeigt sich für die Medienpädagogik nur eine zögerliche Bezugnahme. Im Folgenden wird zunächst das Konzept der Gouvernementalität knapp eingeführt. Daraufhin soll ein Einblick in allgemeinpädagogische Bereiche gewährt werden, die sich bereits ausführlicher mit Gouvernementalität befasst haben, bevor anschliessend ein Blick auf das Feld der Medienpädagogik und deren Rezeption von Gouvernementalität als analytischer Zugang geworfen wird.

\subsection{Grundzüge des Gouvernementalitätsansatzes - Verstrickungen von Macht, Herrschaft und dem Selbst}

Im Zuge seiner Arbeiten zu historisch veränderlichen Formen des Regierens konstatiert Foucault (2012), dass eine Auseinandersetzung mit isolierten Aspekten staatlicher Führung moderne Regierungsformen nicht hinreichend erklären kann. «Regierung> meint für Foucault dabei «die Führung von Menschen im Sinne ihrer Lenkung, 
Kontrolle und Leitung» (Maurer und Weber 2006, 10), wobei sowohl Fremd- als auch Selbstführung gemeint ist. Es ist dieser Zusammenhang von Selbst- und Herrschaftstechniken, der aus gouvernementalitätsanalytischer Sicht im Mittelpunkt steht. Die Führung im Sinne der Ausübung legitimer Herrschaft, die einer politischen Rationalität folgt, umfasst dabei bestimmte Praktiken, «in deren Kontext Wahrnehmungs- und Beurteilungsstrategien generiert werden» (Lemke, Krasmann, und Bröckling 2012, 20). Gouvernementale Regierungsformen können, in anderen Worten, verstanden werden als «Beziehungsnetz von subtilen Zwängen, die sich zusehends der Individuen bemächtigen, indem sie sie zu permanenter Selbstprüfung, Selbstartikulation, Selbstdechiffrierung und Selbstoptimierung anstacheln» (Pongratz 2010, 376).

Foucaults Unterscheidung von Disziplinarmacht und Sicherheitsdispositiven bzw. -technologien stellt einen wesentlichen Aspekt des Gouvernementalitätskonzepts dar. Während Disziplinarmacht für einen Typus der Regierung steht, der «hierarchisierende Trennungen [installiert], die zwischen Ungeeignetem und Geeignetem, Normalem und Anormalem unterscheiden» (Lemke, Krasmann, und Bröckling 2012, 13), sind Sicherheitsdispositive komplementär zu verstehen:

«Die Sicherheitstechnologie repräsentiert das genaue Gegenteil des Disziplinarsystems: Geht dieses von einer (präskriptiven) Norm aus, so ist der Ausgangspunkt des Sicherheitssystems das (empirisch) Normale, das als Norm dient und weitere Differenzierungen erlaubt. Statt die Realität an einem zuvor definierten Soll auszurichten, nimmt die Sicherheitstechnologie die Realität selbst als Norm: als statistische Verteilung von Häufigkeiten, als Krankheits-, Geburten- und Todesraten etc. Die »Dispositive der Sicherheit« ziehen keine absoluten Grenzen zwischen dem Erlaubten und dem Verbotenen, sondern spezifizieren ein optimales Mittel innerhalb einer Bandbreite von Variationen.» (ebd., 13f.)

Insbesondere in den Erscheinungsformen neoliberaler Gouvernementalität, etwa in Form einer Ökonomisierung des Selbst, kommt dieser spezifische Führungstypus zum Ausdruck und hat Einzug in verschiedene gesellschaftliche Teilbereiche gehalten (Bröckling 2007). Die Verbreitung neoliberaler Logiken tritt dabei als Verbindung aus spezifischen Modi der Subjektivierung und Normalisierungstendenzen in Erscheinung. Entscheidend ist dabei, dass diese Modi sich universell manifestieren und die Ökonomie sich nicht länger als spezifisches und umgrenzbares Feld erweist, sondern in die verschiedensten Bereiche der Gesellschaft hineinwirkt und «prinzipiell alle Formen menschlichen Verhaltens [umfasst]» (Lemke, Krasmann, und Bröckling 2007, 16). 


\subsection{Gouvernementalität im erziehungswissenschaftlichen Diskurs}

Sehr früh erkannte Ludwig Pongratz (1988) das analytische Potenzial von Foucaults Arbeiten, etwa für die historische Bildungsforschung, aber auch bezüglich des Dispositivkonzepts als machtanalytische Hintergrundfolie schulbezogener Fragestellungen (Pongratz 1990). Zentrale Übersichtsarbeiten zur Gouvernementalität aus erziehungswissenschaftlicher Perspektive liegen in Form der Sammelbände von Norbert Ricken und Markus Rieger-Ladich (2004) sowie von Susanne Weber und Susanne Maurer (2006) vor. Viel Aufmerksamkeit im Kontext von macht- und herrschaftsbezogenen Fragen gegenwärtiger Gesellschaftsentwicklungen erhielten in der Erziehungswissenschaft auch die Arbeiten zur «Gouvernementalität der Gegenwart» von Ulrich Bröckling, Susanne Krasmann und Thomas Lemke (2012). Die hier vorzufindenden «Studien zur Ökonomisierung des Sozialen» (ebd.) sind gerade deswegen von erziehungswissenschaftlicher Relevanz, weil sie unter einer von Foucaults Arbeiten angeleiteten Perspektive Entgrenzungsphänomene dechiffrieren, die die Veränderung der Verhältnisse sozialstaatlicher Institutionen, politischer Rationalitäten und ökonomischer Verwertbarkeitslogiken nachzeichnet. Hilfreich ist diese Perspektive insbesondere, da sie einen Rahmen bietet, ein breites Spektrum von Veränderungen im 〈Feld der Bildung) (angefangen bei transnationalen Hochschulreformen über Fragen der Professionalisierung bis hin zu Umbauten schulischer Curricula) analytisch zu durchdringen und in einen gesamtgesellschaftlichen Kontext einzuordnen. Zudem ist die Zentralstellung des macht- und herrschaftsbezogenen Instrumentariums des Gouvernementalitätsansatzes prädestiniert dafür, an pädagogische Fragestellungen anzuschliessen, da diesen ein solcher Impetus immanent ist. Mit anderen Worten: Pädagogische Beziehungen sind per se machtförmig und konstitutiv an die Aushandlung normativer Setzungen und entsprechender Positionierungen gebunden. Diese Prozesse sind stets situiert und sozio-kulturell präformiert, wodurch sich für eine erziehungswissenschaftliche Perspektive die Herausforderung ergibt, diese Präfiguration einzuordnen und jene Spielräume zu eröffnen, in denen ein «Sich-Nicht-Regieren-Lassen» (Maurer und Weber 2006, 29) möglich wird.

Die Grundidee der von Foucault vertretenen These der Hinwendung zu sanften Formen der Disziplinierung bricht Pongratz $(2009,188)$ wie folgt herunter: «Stets geht es darum, Sichtbarkeiten und Sagbarkeiten herzustellen». Bezogen auf Bildungszusammenhänge verbirgt sich hinter Schlagworten wie individualisiertes), (eigenverantwortliches〉, ‘selbstgesteuertes) und «lebenslanges Lernen〉 eine gouvernementale Programmatik als Ausdruck neuer Regierungslogiken, welche in Form eines auf Prävention ausgelegten (Risikomanagements) Verantwortung entstaatlicht und stattdessen das sich selbst beobachtende und reflektierende Subjekt zur Kontrollinstanz über den eigenen Lernerfolg erhebt. Die Herstellung von Sichtbarkeit und Sagbarkeit ist damit eng an die Lernenden selbst und deren Fähigkeit zur Verinnerlichung der entsprechenden Praktiken gebunden, die als erfolgsversprechend gelten. 
Für Pongratz nimmt die Pädagogik im Kontext gouvernementaler Programmatiken einen besonderen Stellenwert ein, da sie aufgrund ihrer charakteristischen subjektivierenden Zielrichtung einen wesentlichen Beitrag zur Verankerung ebensolcher Formen der Selbstführung leisten kann:

«Im Zuge dieser Gewichtsverlagerung gewinnt Pädagogik eine immense Bedeutung: Schule und Weiterbildung, Erziehungseinrichtungen und Sozialarbeit werden eingebunden in einen strategischen Komplex, der darauf abzielt, die gesellschaftlichen Verhältnisse auf der Grundlage einer neuen Topographie des Sozialen zu recodieren» (ebd., 191).

\subsection{Medienpädagogische Rezeptionslinien der Gouvernementalität}

Während gouvernementalitätsanalytische Arbeiten in verschiedenen Bereichen der Erziehungswissenschaft mittlerweile zu einem etablierten Zugang avanciert sind (siehe exemplarisch Forneck und Wrana (2005) zur Erwachsenenbildung; Anhorn, Bettinger und Stehr (2007) zur sozialen Arbeit; Ntemiris (2011) zu Kindheit; Dzierzbicka (2006) zur Schule), schliessen Autorinnen und Autoren im subdisziplinären Feld der Medienpädagogik eher selten an diese Entwicklungen an (als Ausnahmen siehe z. B. Lang 2013; Neurath 2017; Dander 2018). Erstaunlich erscheint dies unter anderem deshalb, weil die Bedeutung von Medien für Fragen moderner Formen von Macht und Herrschaft und die daran gekoppelten Technologien des Selbst vonseiten gouvernementalitätsanalytischer Arbeiten (ohne dezidiert erziehungswissenschaftlichen Bezug) durchaus regelmässig betont werden (bspw. Stauff 2005; Kauppinen 2012; Skrandies 2014; Rieger 2018). Angesichts des medienkulturellen Wandels der letzten Jahre, der unter anderem durch Schlagwörter wie «Social Media) oder ‘Partizipation ) und (Openness) geprägt war bzw. ist, wird digitalen Medien zunehmende Relevanz für Prozesse der Geltendmachung von Wahrheit zuerkannt. Folgt man der These einer fortgeschrittenen digital-medialen Konstitutivität der Gegenwartsgesellschaft, die etwa durch Konzepte wie die rechnerische Konstruktion sozialer Wirklichkeit (Seyfert und Roberge 2017) oder die hegemoniale Position datenbasierter Plattformen der «Big-Tech»-Unternehmen für Fragen algorithmisch präfigurierter Subjektivität (Cheney-Lippold 2017) beschrieben werden kann, wird deutlich, dass digitale Medialität und Gouvernementalität eng miteinander verzahnt sind. Hierbei werden dezidiert Machtfragen ins Spiel gebracht, die im Zusammenhang mit der Etablierung neuer (algorithmisierter) Rationalitäten stehen. Diese legen die Frage nahe, inwiefern wir es mit einem neuen Typus der Verbindung von Herrschafts- und Selbsttechniken - sprich: einer neuen Form der Gouvernementalität - zu tun haben, die im Zusammenspiel mit dem digital-medialen Strukturgefüge veränderte Technologien des Selbst zeitigt. In diesem Sinne hält bspw. John Cheney-Lippold mit Verweis auf den von der Philosophin Antoinette Rouvroy geprägten Begriff der ‘algorithmic 
governmentality fest: «Who we are in the face of algorithmic interpretation is who we are computationally calculated to be» (Cheney-Lippold 2017, 5). Hinzu kommt, dass auch in bildungspolitischer Hinsicht in den letzten Jahren zahlreiche Entwicklungen verzeichnet werden können, die dezidiert das medienpädagogische Feld betreffen. Exemplarisch lassen sich für Deutschland die Papiere der Kultusministerkonferenz (KMK) zur «Medienbildung in der Schule» (2012) sowie zur «Bildung in der digitalen Welt» (2017) heranziehen, in Österreich die Initiative zur «digitalen Grundbildung» ${ }^{10}$ und für die Schweiz der «Lehrplan 21» ${ }^{11}$. Die Schaffung dieser Rahmungen und diese konkreten Reformen teilen den gemeinsamen Ausgangspunkt, eine angemessene Reaktion des Bildungssystems auf Digitalisierungsprozesse bieten zu wollen. Insofern haben sie weitreichende Konsequenzen für die Positionierung der Bildungsinstitutionen und der darin tätigen Akteurinnen und Akteure hinsichtlich der Frage nach der Festschreibung von Rahmenbedingungen für Subjektivierungsweisen im Kontext der Digitalität.

Im Folgenden soll es darum gehen, am Beispiel des $21^{\text {st-Century-/4K-Skills-Dis- }}$ kurses Formen und Figuren ebensolcher Bezugnahmen auf Digitalität und Bildung vor dem Hintergrund gouvernementaler Strategien darzulegen und aus erziehungswissenschaftlicher Perspektive einzuordnen.

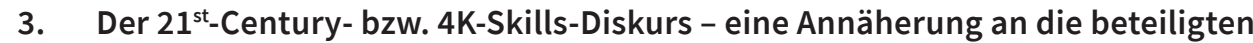 Akteurinnen und Akteure}

Angesichts der oben entfalteten Überlegungen erfolgt im nächsten Schritt eine gouvernementalitätsanalytisch inspirierte Betrachtung des $21^{\text {st-Century-/4K-Skills- }}$ Diskurses. Hierzu wird in einem ersten Schritt das Phänomen skizziert und die für diesen Komplex konstituierenden Hintergründe betrachtet. ${ }^{12}$ Der Entstehungszu-

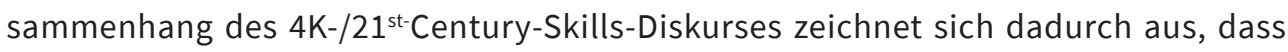
er unterschiedliche Ebenen umfasst, indem er als institutioneller Wandlungsprozess, als inhaltlich-curriculare Setzung (realisiert in einem spezifischen Set pädagogischer Praktiken) sowie als bildungspolitische Rahmung in Erscheinung tritt. Niederschlag findet dieser Komplex in Form von Publikationen, Tagungen und Vorträgen, der Gründung von Bildungs- und Beratungsorganisationen und der Initiierung von Strukturund Curriculumsreformen bis hin zu Lehr- und Lernmaterialien. Besonders sticht das immer wieder zitierte Rahmenmodell der $21^{\text {st }}$-Century-Skills hervor, dessen zentrales

10 https://www.bmbwf.gv.at/Themen/schule/zrp/dibi/dgb.html.

11 https://www.lehrplan21.ch/.

12 Im Sinne einer Vorbemerkung sei an dieser Stelle darauf hingewiesen, dass die Rede von einem politischpädagogischen Macht-Wissen-Komplex ökonomische Rationalitäten einschliesst. Mit Simons $(2004,166)$ wird davon ausgegangen, dass «in der neoliberalen Regierungskonfiguration der Unterschied zwischen der Ökonomie und dem Sozialen als bislang zwei sauber voneinander getrennten Bereichen keine Bedeutung mehr [hat]». 
Element die 4K-Skills sind. Das erklärte Ziel des Rahmenmodells ist die Beschreibung von Fähigkeiten und Fertigkeiten, deren Erwerb als entscheidend für persönlichen und beruflichen Erfolg der Lernenden dargestellt wird («This Framework describes the skills, knowledge, and expertise students must master to succeed in work and life» (P21 2019, 2)). Das Rahmenmodell bildet das Fundament für die intendierten Veränderungen von Bildungsinstitutionen und richtet sich dabei auf unterschiedliche Ebenen und Akteurinnen und Akteure.

Massgeblich befördert wurde die 4K-Skills-Initiative durch das Center for Curriculum Redesign (CCR), das sich als «unabhängige, unparteiische, internationale Organisation» (Fadel, Bialik, und Trilling 2017, 67) bzw. als «internationales Gremium und Forschungscenter, das danach strebt, die Möglichkeiten der Menschheit zu vergrössern und den gemeinsamen Wohlstand zu verbessern» (ebd., 191), bezeichnet. Im «Foundational White Paper» (Fadel 2015) wird die Rolle des CCR als vernetzende Organisation beschrieben, die in globalem Massstab «non-governmental organizations, jurisdictions, academic institutions, corporations, and non-profit organizations including foundations» (ebd.) zusammenbringt, um weitreichende Bildungsreformen voranzubringen, die besonders auf die Frage zukunftsrelevanter Inhalte abzielen. Als beteiligte Organisationen und Unternehmen werden unter anderem die OECD, die UNESCO, die Asia Society sowie die World Bank Group neben Tech-Unternehmen wie Google, Microsoft und IBM angeführt. ${ }^{13}$

Daneben ist das Netzwerk Partnership for 21st Century Skills zu nennen, das seit 2018 der Organisation Battelle for Kids angehört, einer «national, not-for-profit organization with the mission of realizing the power and promise of $21^{\text {st }}$ century learning for every student». ${ }^{14}$ Auch hier spielt die Absicht einer fundamentalen curricularen Reform des Bildungssystems die zentrale Rolle: «Our mission is to realize the power and promise of $21^{\text {st }}$ century learning for every student - in early learning, in school, and beyond school - across the country and around the globe». ${ }^{15}$ Ein Blick auf die treibenden Kräfte des P21-Netzwerks offenbart einen Zusammenschluss USamerikanischer Organisationen und Unternehmen (bspw. der Disney-Konzern, die American Camp Association, Amplified IT (eine auf den Bildungsbereich spezialisierte Beratungsagentur), CatchOn (ein Educational-Data-Mining-Unternehmen)). Sehr offensiv wirbt auch der unter anderem für die Koordination der PISA-Studie zuständige OECD-Bildungsdirektor Andreas Schleicher für einen Umbau des Bildungssystems gemäss den $21^{\text {st }}$-Century-Skills. ${ }^{16}$ Gestützt und verbreitet werden die Grundideen zudem durch zahlreiche weitere global agierende Netzwerke und Organisationen,

13 https://curriculumredesign.org/partners/.

14 https://www.battelleforkids.org/about-us.

15 https://www.battelleforkids.org/networks/p21.

16 http://www.oecd.org/general/thecasefor21st-centurylearning.htm. 
wie etwa die Global Partnership for Education ${ }^{17}$, die sich schwerpunktmässig auf Bildungsreformen in einkommensschwachen Ländern spezialisiert hat, oder dem Netzwerk Education Reimagined, das im US-amerikanischen Kontext operiert und ebenfalls grundlegende Transformationen des Bildungssystems anstrebt, mit dem Ziel, "to powerfully serve, include, value, and love every single child». ${ }^{18}$ Insgesamt findet sich ein kaum zu durchschauendes Netzwerk unterschiedlichster Gruppierungen sowie Akteurinnen und Akteure, das dem $21^{\text {st }}$-Century-/4K-Skills-Diskurs mit grosser Stimmgewalt Sichtbarkeit verschafft und keinen Zweifel daran lässt, dass die angestrebten Reformen auch so umfassend gemeint sind, wie es den Anschein hat.

\section{Gouvernementale Strategien im globalen Spielfeld von Digitalisierung und Bildung}

Bereits ein erster Blick in zentrale Dokumente des $4 \mathrm{~K}-/ 21^{\text {st }}$-Century-Skills-Diskurses offenbart einige interessante Aspekte: Zunächst fällt an zahlreichen Passagen ein sprachlicher Duktus auf, der einen deutlichen Aufforderungscharakter aufweist und besonders dahingehend klare Forderungen an Lehrende und Lernende richtet, wie sie zu sein haben, was sie wissen und was sie können müssen. Formulierungen wie "Think creatively», «Work creatively with others», «Reason effectively», "Create media products» usw. (P21 2019, 4ff.) lassen sich als exemplarische Fundstellen für ein wiederkehrendes Muster heranziehen, das unmissverständlich das Vorhandensein einer ganz bestimmten Vorstellung eines idealtypischen Lernenden-Subjekts als Zielvorstellung verdeutlicht. Ein wiederkehrendes Leitmotiv von Vertreterinnen und

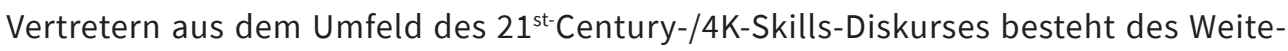
ren in der Argumentationsfigur einer aufgrund globaler Veränderungen unabdingbaren Notwendigkeit der umfassenden Neujustierung von Bildungszielen. Dementsprechend umfangreich gestalten sich zeitdiagnostische Erörterungen, (siehe z. B. Sterel, Pfiffner, und Caduff 2018, 17-87 oder Fadel, Bialik, und Trilling 2017, 9-35), wobei die hierbei dargelegte globalgesellschaftlichen Transformationen der Gegenwart als argumentativer Anker für einen Veränderungsdruck herangezogen werden, der sich sowohl für Individuen als auch Institutionen und ganze Staaten ergebe und über alle Gesellschaftsbereiche hinweg eine Reaktion erfordere. Betrachtet man die Konstruktionen um diesen zentralen und sich durch nahezu alle Dokumente ziehenden Aspekt, werden (in dieser Reihenfolge) z. B. «Die Veränderung der Wirtschaft», «Digitalisierung», «Kooperation», «Freiheit und Autonomie» sowie «die postmoderne Befindlichkeit» (Sterel, Pfiffner, und Caduff 2018, 5) als Aspekte genannt, welche bestehende gesellschaftliche Institutionen, aber auch individuelle Orientierungen fragil werden lassen. In diesem Kontext kommt dem Bildungssektor eine besondere

17 https://www.globalpartnership.org/.

18 https://education-reimagined.org/about/. 
Rolle zu, da dieser einerseits unter besonders grossem Veränderungsdruck stehe, andererseits diesem Bereich aber auch eine Schlüsselfunktion zur gesellschaftlichen Problemlösung zugeschrieben wird. Das übergreifende Ziel des $4 \mathrm{~K}-/ 21^{\text {st }}$-CenturySkills-Diskurses besteht im Anschluss daran in der unbedingten Forderung einer Neuausrichtung des Bildungssystems im Sinne der vorgelegten Bildungsziele. Das erklärte Ziel ist somit die Umsetzung eines «holistic approach to delivering the educational experience and environment that every student needs to be a lifelong learner and contributor» (Battelle for Kids 2020, 9). Als Antwort werden Kriterienkataloge präsentiert, die ein Set an handlungsleitenden Orientierungen vorschlagen, wonach sich Bildungsinstitutionen sowie Lehrende und Lernende zu richten haben, bis hin zur Vorgabe, welche Fächer als relevant gelten. Mit Nachdruck und in universalisierender Manier wird das Erreichen dieser Ziele als (einziger) Weg beschrieben, um als Individuum wie auch als Gesellschaft erfolgreich zu sein. Deutlich wird dies schon im Untertitel der Originalausgabe des Buches von Fadel, Bialik und Trilling (2017), die mit Blick auf die Herausforderungen des 21. Jahrhunderts von «Competencies Learners Need to Succeed» sprechen.

Im Folgenden werden analytisch zentrale Figuren des Diskurses unter den Gesichtspunkten der Formen von Selbst- und Fremdführung sowie den damit verbundenen Normen und Normalisierungen pointiert.

\subsection{Formen der Selbst- und Fremdführung ...}

Eine zentrale und ebenso paradox anmutende Figur, die sich konstant durch den $21^{\text {st. }}$ Century-/4K-Skills-Diskurs zieht, besteht darin, dass Lernende für eine Zukunft ausgebildet werden, die sie durch die Ausrichtung ihrer Ausbildung an den Prämissen der $4 \mathrm{~K}-/ 21^{\text {st }}$-Century-Skills überhaupt erst erzeugen. Der Diskurs argumentiert hierbei mit einer präventiven Logik: Es geht um das rechtzeitige Ergreifen von Massnahmen zur Verhinderung des ‘Abgehängt-Werdens` in einer nahenden Zukunft, deren Anforderungen - so zumindest die dabei implizit vertretene Annahme - sich unmittelbar aus der Gegenwart ableiten lassen. Gerahmt wird diese Logik der Vorbeugung nicht nur durch ein Bedrohungsszenario, das die Gefahr individuellen und gesellschaftlichen Scheiterns miteinander verschränkt, sondern auch durch ein Erfolgsnarrativ in Form von emanzipatorischer Selbstentfaltung. Es fällt auf, dass hierbei eine «Gefahr des Scheiterns) im Sinne des ausbleibenden beruflichen Erfolgs (der teils gleichgesetzt wird mit einer umfassenderen Idee von einem «misslungenen Leben`) als impliziter Gegenhorizont konstruiert wird. Zur Vermeidung dieses Bedrohungsszenarios wird eine Norm der individualistischen Konformität nahegelegt, die ihren Ausdruck darin finden soll, dass die lernenden - bzw. hilfsbedürftigen - Subjekte erst durch gesteigerte Anpassungsfähigkeit an äussere Anforderungen in die Lage versetzt werden, mündige und freiheitliche Subjekte zu werden. «Adapt to change» (P21 2019, 6) 
erweist sich dabei nicht nur als Floskel, sondern als Kerngedanke, der hier in Gestalt eines Bildungszieles formuliert wird. In diesem Sinne hält auch der OECD-Bildungsdirektor, Andreas Schleicher, fest: «Success will go to those individuals and countries that are swift to adapt, slow to resist and open to change». ${ }^{19}$

Formeller Bildung wird dabei eine gesellschaftliche Schlüsselrolle zuteil, indem sie die zentrale Schaltstelle für Präventivmassnahmen darstellt, um im Sinne von «Dispositiven der Vorbeugung» (Bröckling 2017, 96) die Gefährdungspotenziale durch gegenwärtige gesellschaftliche Umbrüche rechtzeitig zu minimieren. Individualisierung und Universalisierung erweisen sich hierbei als eng miteinander verbunden, indem die Ausrichtung der individuellen Lebensführung entlang der normativen Leitlinien ihre Legitimation dadurch erhält, dass sie im Dienste globaler Optimierungsmassstäbe steht. Die dabei adressierten ethischen Grundsätze, die auf Aspekte wie Steigerung der Lebensqualität, Gleichheit und Gemeinwohl rekurrieren, lassen sich durch ihre Universalität kaum als Ziele infrage stellen. Fraglich bleibt aber der unterstellte Kausalzusammenhang zwischen dem Erreichen dieser Ziele durch Übernahme der vorgeschlagenen Programmatik.

Wiederkehrend zeigt sich die Argumentation, dass die skizierten Problemlagen nur durch globale Zusammenschlüsse gelöst werden können und nicht exklusiv durch pädagogische oder politische Zugänge. Pädagogische und ökonomische Expertise sowie die jeweiligen Interessenlagen werden dabei als gleichrangig verstanden, eventuelle Zielkonflikte, die in einer solchen Konstellation entstehen können, werden nicht thematisiert. Ganz im Gegenteil werden Zielvorstellungen und Absichten stark homogenisiert und angesichts einer sich scheinbar als offensichtlich erweisenden Schieflage eines ebenfalls gleichförmig erscheinenden weltweiten Bildungssystems als einzig gangbarer Weg dargestellt.

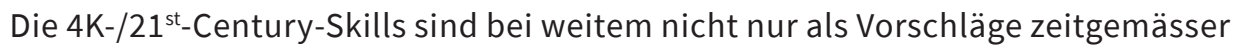
Lernziele zu verstehen. Vielmehr handelt es sich um eine Etablierung umfassender Steuerungsinstrumente, die eine Variante eines gouvernementalen Führungstypus repräsentieren, der aus Analysen neoliberaler Umbauten des Bildungssystems bekannt ist (bspw. Maurer und Weber 2006; Pongratz 2009). Insofern finden sich auch hier die - etwa mit Blick auf die Bologna-Reform typischen - Formen von Selbstführung als Zielwert lernender Subjekte. Diese Formen sind im hier betrachteten Zusammenhang jedoch als noch umfassendere Modelle der individuellen Lebensführung konzipiert, indem sie weit über den Bereich institutionalisierter Bildung hinaus gehen und ein Set grundlegender Orientierungen, geknüpft an den Erwerb von Techniken der Selbstbeobachtung und -kontrolle, als ebenso erstrebenswertes wie alternativloses Ziel beschreiben. Trägt man dem Umstand Rechnung, dass die notwendigen Instrumente des Leistungsvergleichs im Bildungssektor bereits implementiert wurden - man denke an die PISA-Studie oder die zahlreichen Universitätsrankings - wird

19 http://www.oecd.org/general/thecasefor21st-centurylearning.htm. 


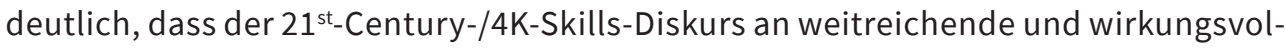
le Steuerungsmechanismen andockt und diese zugleich legitimiert, indem sie implizit als Generatoren einer empirischen fundierten Norm anerkannt werden, deren Evidenzbasierung Wahrheiten und Bedarfe aufdeckt und somit Reformen legitimiert.

Zur Logik der Prävention tritt dabei ausserdem eine Progressionslogik, wodurch die so konzipierte Position nicht nur als eine Strategie der Absicherung gegenüber einem drohenden Risiko des (Abgehängt-Werdens) markiert wird, sondern auch als optimaler Weg einer als erstrebenswert dargestellten Erfolgsgeschichte, in der ökonomischer Wohlstand, individuelles Lebensglück und Allgemeinwohl als widerspruchsfreies Ensemble realisiert werden. Individuelle und kollektive Bedürfnisse fallen dabei in eins, eine klare Unterscheidung der Zielsetzung für Staaten, Organisationen und Individuen scheint angesichts einer solchen Adressierung eines Kollektivsubjekts (Alkemeyer, Bröckling, und Peter 2018) obsolet. Selbstermächtigung scheint dabei per se im Dienste einer globalistisch-ökonomischen Rationalität verstanden zu werden. Entscheidend ist, dass Absicherung zur Aufrechterhaltung eines Status Quo nicht das alleinige Ziel darstellt. Vielmehr geht es um eine Optimierungstrategie angesichts einer spezifischen Zukunftserwartung, die ähnlich wie eine «Fiktion der wahrscheinlichen Realität» (Esposito 2007) mit Szenarien operiert, die auf Grundlage der Beobachtung der Gegenwart Gestaltungszwänge generiert und zugleich ein hohes Mass an Steuerbarkeit annimmt. Die vorgeschlagenen Eingriffe in das Bildungssystem bis hinein in die Bildungsinhalte, bei denen auch nicht-staatliche Akteurinnen und Akteure gleichermassen beteiligt werden sollen, erscheinen dabei nicht nur als legitim, sondern geradezu als obligatorisch.

Es deutet sich an, dass die im Diskurs durchscheinende De-Thematisierung der Rolle von Hochschulen bzw. Universitäten auch als institutionelle Entgrenzung gedeutet werden kann, welche in Gestalt der Frage erscheint, wer berechtigt ist, derlei inhaltliche Setzungen im Bildungsbereich vorzunehmen, was als moralische Verpflichtung in Szene gesetzt wird. In diesem Sinne schreiben Fadel, Bialik und Trilling: «Wir sind davon überzeugt, dass jeder von und in der Verantwortung steht, das Bestmögliche bei der Gestaltung unseres Bildungswesens zu tun, selbst angesichts von Unsicherheiten» $(2017,182)$. Im Anschein der Etablierung partizipativer Strukturen werden sodann weitreichende Entscheidungsbefugnisse im Bildungswesen als diffuses Gemeingut stilisiert, wobei die Spielräume präskriptiv gesetzt werden und dabei Leitlinien, bspw. Anpassungsfähigkeit (der Lernenden selbst als auch der Bildungsinstitutionen) vorausgesetzt wird. 


\section{2 ... im Zusammenhang von Normen und Normalisierungen}

Die für gouvernementale Führung essenziellen Bereiche der Sicherheit sowie der Disziplin zeichnen sich - wie oben bereits angesprochen - durch jeweils unterschiedliche Normalisierungstechniken aus. Während die Logik der Disziplin darin besteht, ein «optimales Modell zu setzen, das in bezug auf ein bestimmtes Resultat konstruiert ist» (Foucault 2019, 89) und damit eine präskriptive Setzung zur Ausrichtung des Handelns vollzogen wird, die eine Unterscheidung zwischen Normalem und Anormalem erlaubt, nimmt die Logik der Sicherheit eine Normalität als Ursprungszustand an, von der aus Normalisierung darin besteht,

«Normalitätsaufteilungen wechselseitig in Gang zu setzen und auf diese Weise zu bewirken, daß die ungünstigsten auf die günstigsten zurückgeführt werden. [...] Das Normale kommt als erstes, und die Norm leitet sich daraus ab, oder die Norm setzt sich ausgehend von dieser Untersuchung der Normalität fest und spielt ihre operative Rolle» (ebd., 98).

Mit Blick auf den hier herangezogenen Gegenstand lassen sich sowohl Normalisierungsstrategien erkennen, die einer Disziplinarlogik folgen und eine orientierende Setzung vornehmen, als auch solche Strategien, die in umgekehrter Weise Normalität über empirische Zustandsbeschreibungen ergründen, um dann entsprechende regulatorische Mechanismen zu implementieren, die helfen, ein so definiertes Optimum zu erreichen. Die sich durch den $21^{\text {st }}$-Century-/4K-Skills-Diskurs ziehenden Eigenschaftszuschreibungen eines optimalen Lernenden-Subjekts, die mit konkreten zu erreichenden Zielsetzungen einhergehen und bis hin zur Formung eines bestimmten Charakters reichen, sowie die Setzung eines Sets bestimmter Lernziele und Themen ( Moderne Themen wie Robotik, Entrepreneurship, Programmieren und Medienkompetenz müssen eingeführt werden» (Fadel, Bialik, und Trilling 2017, 54)) lassen sich als Führungsformen verstehen, deren normalisierender Gehalt in einer Disziplinarlogik zum Ausdruck kommt. In ihrer (proklamierten) Ausführung jedoch finden sich Anhaltspunkte, die stärker an Normalisierungsstrategien der Sicherheitstechnologien angelehnt sind, indem der Schwerpunkt nicht auf dem Sanktionieren von Abweichung liegt, sondern in erster Linie vorbeugende Praktiken etabliert werden sollen. Der beschriebene Zusammenhang lässt sich damit als «Regime der Prävention» (Bröckling 2017, 96) beschreiben, die «aus Wissens- und Rechtfertigungsordnungen» (ebd., 97) bestehen, dabei "spezifische Verfahren und institutionelle Arrangements» (ebd.) umfassen und schliesslich «mit Adressierungen und Selbstdeutungsmustern, affektiven Dispositionen und Konzepten von Agency» (ebd.) verbunden sind und somit Subjektpositionen zuweisen.

Die Normalisierungsstrategien und -techniken im Zusammenhang des $21^{\text {st }}$ Century-/4K-Skills-Diskurses erscheinen als Versuche, einen unmittelbar drohenden Verlust von Lebensqualität aufgrund einer sich unumkehrbar verändernden 
Gesellschaft zu minimieren. Die Deutung dieser Gefahr als Risiko ermöglicht es, «vorbeugende oder schadensausgleichende Maßnahmen» (ebd., 86) zu treffen, um durch bestmögliche strategische Züge die drohenden Entwicklungen schon frühzeitig abzuwenden. Es lässt sich feststellen, dass nicht nur ein Wissen um zukünftige Anforderungen im hier betrachteten Diskurs behauptet wird, sondern dezidiert umfassende Veränderungsstrategien vorgelegt werden, die (bildungs-)institutionelle Settings auf diese Anforderungen ausrichten sollen. Ein wichtiger Bestandteil dieser auf Prävention ausgelegten Steuerungsmechanismen sind die, sich selbst immer wieder transparent machenden (individuell sowie kollektiv verstandenen) Subjekte, deren «Bildungserfolge` von der Fähigkeit einer möglichst umfassenden Anpassung an das vorgelegte Set von ‘Skills〉 abhängen. Die legitimatorische Basis erhält diese Zurichtung durch eine doppelte moralische Fundierung, da in der hier zugrunde gelegten Logik suggeriert wird, dass nicht nur die individuelle Existenzsicherung gewährleistet, sondern durch die Aneignung der vorgelegten Eigenschaften zudem ein grösserer gesellschaftlicher Auftrag erfüllt wird. Fundiert wird dies auch durch die Orientierung an einer empirisch generierten Normerwartung, z. B. durch LargeScale-Assessments (siehe bspw. der Report von Global Partnership for Education 2020) ${ }^{20}$, die Handlungsbedarfe als evidenzbasierte Notwendigkeit darstellt. Die so erhoffte Risikominimierung zur Gefahrenabwehr kommt dabei in Form der Erfüllung allgemeinwohlorientierter Zielsetzungen daher, die durch rechtzeitiges Ergreifen von Präventionsmassnahmen erlangt werden kann und ihren normalisierenden Charakter in der Erhaltung von Wohlstand und der Steigerung von Lebensqualität durch Fügung in das vorgeschlagene Subjektivierungsschema entfaltet.

Die damit einhergehende Reproduktion einer Norm der Wettbewerbsorientierung im Bildungssystem (Höhne 2015), die in Gestalt einer umfassenden Legitimierung ökonomischer Rationalitäten im Diskurs zutage tritt und unter der Prämisse der Vereinheitlichung sowohl auf inhaltlicher als auch systemischer Ebene eine grundlegende Neuorientierung einfordert, setzt wesentlich an der Implementierung von Kontrollsystemen an. «Standardisierung und Assessment» (Fadel, Bialik, und Trilling 2017, 66) sind die Mechanismen, durch die man sich erhofft, dass «Veränderungen auf diesen beiden Ebenen sich letzten Endes auf alle Ebenen durchschlagen werden» (ebd.). Nahezu selbstverständlich reiht sich die damit verbundene Position in Entwicklungen ein, die schon vor einigen Jahren in der diskursprägenden Etablierung des Qualitätsbegriffes im Feld des Pädagogischen als Merkmal einer Ökonomisierung des Bildungsbereichs zum Ausdruck kam (Höhne 2015, 61-63). Wettbewerbsorientierung und das damit verbundene Effizienzstreben lassen sich sodann als Wegbereiter verstehen, durch die die Voraussetzungen geschaffen werden, den erzeugten Bedarf nach inhaltlichen Richtlinien der Bildungssteuerung zu befriedigen. Durch die so

20 https://www.globalpartnership.org/sites/default/files/document/file/2020-01-GPE-21-century-skills-report.pdf. 
zum Ausdruck gebrachte Beanspruchung der Definitionshoheit über Erfolgskriterien von Bildung wird eine regulatorische Norm erzeugt, die losgelöst von institutionellen Voraussetzungen oder staatlichen Bedingungen Geltungsanspruch erhebt.

\section{5. (Medien-)Pädagogische Implikationen}

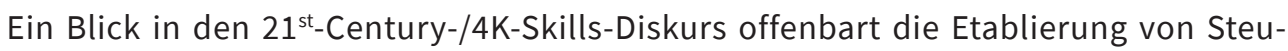
erungstechnologien, deren Ursprünge im Bildungsbereich liegen, wobei die Zielsetzung jedoch weit darüber hinaus geht. Markant ist der kaum durchschaubare politisch-ökonomische Komplex, der hinter den hier beschriebenen Phänomenen steht und die Anliegen mit grosser Deutlichkeit in vielgestaltiger Form vorträgt. Die exemplarische Bezugnahme auf die hier herangezogenen Diskursfragmente repräsentiert nur einen kleinen Ausschnitt aus einem komplexen global vernetzten Gefüge, deren massgebliche Initiative sich aus bildungspolitischen Akteurinnen und Akteuren sowie einer Vielzahl an Wirtschaftsunternehmen sowie verschiedensten NGOs speist. Es zeichnet sich insgesamt ab, dass wir es mit einer Variante neoliberaler Regierungsformen zu tun haben, die im Sinne eines «Netzwerks der Politikgestaltung» (Raithelhuber 2006) in Erscheinung tritt.

Auffällig ist die Suggestion von Eindeutigkeit der vorgelegten Gegenwartsdiagnose, deren Kernaussage auf der Annahme eines tiefgreifenden medienkulturellen Wandels beruht, welcher umfassenden Veränderungsdruck erzeuge. Anstatt die Unvorhersagbarkeit zukünftiger Anfordernisse anzunehmen, wird eine Position erkennbar, die deutlich die Absehbarkeit künftig notwendiger future skills) betont. Es geht also um Kontingenzminimierung durch eine produktive Wendung der Anforderungsstrukturen einer digital geprägten Gegenwart und der festen Erwartung einer ebenso geprägten Zukunft. Mit der Betonung der inhaltlichen Ebene des Lernens weist der

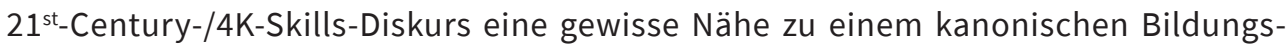
verständnis auf. Dies steht scheinbar im Widerspruch zu der selbst auferlegten Forderung, ein zeitgemässes Bildungsverständnis darlegen zu wollen. Dabei wird deutlich, dass die so angestrebte Selbstentfaltung einer Form der sanften Führung entspricht, die bereits bei früheren Umbauten des Bildungssystems vorherrschende Massgabe war (Pongratz 2004). Neu ist hier jedoch sowohl die auffallend deutliche Beanspruchung von Definitionsmacht über die Setzung von Bildungszielen durch nichtstaatliche Akteure aus der Privatwirtschaft als auch die eindringliche Betonung des Veränderungsbedarfs aufgrund einer im Wesentlichen durch digitale Technologien bedingten gesellschaftlichen Transformation.

Eine dabei zum Ausdruck gebrachte Annahme besteht in der umfassenden Formbarkeit des Menschen, bis hin zu charakterlichen Dispositionen, die als beeinflussbare «Prädiktoren für den weiteren Lernerfolg, für produktive Arbeit und Karriere und für die aktive Übernahme gesellschaftlicher Verantwortung» (Fadel, Bialik, und Trilling 
2017, 147) gesehen werden. Im Sinne eines Trainings bestimmter Eigenschaften, z. B. «Achtsamkeit», «Neugier», «Mut» oder «Resilienz» (ebd. 150), sollen die Voraussetzungen geschaffen werden, in Anbetracht gegenwärtiger Herausforderungen nicht nur zu bestehen, sondern produktive und zukunftsgerichtete Wirksamkeit entfalten zu können. Der hierbei vorgelegte Kriterienkatalog, der im Sinne eines Substrats aus verschiedenen Frameworks konzipiert wurde (ebd., 148f.), wird explizit als präskriptive Norm im Sinne einer Disziplinartechnologie (Foucault 2019 89ff.) verstanden, die «in ihrer zeitgenössischen Gestalt alle negativen Konnotationen wie Sanktion, Drohung, Bestrafung etc. abzuschütteln [versucht]»(Pongratz 2004, 246). Die Aufwertung des Feldes der Pädagogik geht dabei einher mit der Entmächtigung der dieses Feld bisher strukturierenden (Bildungs-)Institutionen, Strukturen und Akteurinnen und Akteuren (z. B. bildungspolitische Gremien und gesetzliche Rahmungen), denen mangelnde Flexibilität unterstellt wird, um sich auf die als notwendig erachteten Präventionsmassnahmen einzustellen. Der von Marttila $(2014,258)$ beschriebene Wandel von einer proaktiven hin zu einer reaktiven Bildungspolitik, die «sich stets am neuesten Stand der empirischen Erkenntnisse orientiert und die bisherigen Handlungsweisen entsprechend des evidenzbasierten Urteils über erreichte Ergebnisse modifiziert», scheint hier nicht zu greifen. Vielmehr lässt sich der hier betrachtete Zusammenhang als Nebeneinander sowohl proaktiver als auch reaktiver Logiken beschreiben. Diese kommen in Gestalt einer Zukunftsgerichtetheit zum Ausdruck, die sich sowohl aus einer evidenzbasierten Ableitung von Bedarfen ergibt als auch in der Absicht zum Ausdruck kommt, durch Setzung übergreifender Rahmenkonzepten progressiv-gestaltend zu wirken.

Betrachtet werden muss das hier diskutierte Phänomen auch im Lichte des zunehmenden Engagements grosser IT-Konzerne im Bildungsbereich. Hier zeichnet sich eine Expansionsstrategie ab, die unter dem Deckmantel philanthropischen Engagements Zugang zu bislang noch nicht erschlossenen Marktbereichen erlangen möchte (Mehta, Creely, und Henriksen 2020, 366ff.). Die Grundlage dieser Entwicklungen stellt auch eine «Affinität zwischen digitalen und kapitalistischen Strukturprinzipien» (Niesyto 2017, 18) dar, die etwa in der Mess- und Berechenbarkeit von Prozessen sowie in Formen der Flexibilitätssteigerung und Modularisierung zum Ausdruck kommt. Ein zentrales Merkmal des im 21 $1^{\text {st }}$-Century-/4K-Skills-Diskurs als Zielrichtung hervorgebrachten Subjekttypus besteht in der Aneignung der Fähigkeit, produktive Verwertbarkeit im Kontext solcher digital-ökonomischen Strukturgefüge zu erkennen und nutzbar zu machen. Kontextualisiert man diese Überlegungen etwa vor dem Hintergrund der Entstehung einer neuen ökonomischen Ordnung, die als «Surveillance Capitalism» (Zuboff 2019) ganz wesentlich menschliche Erfahrungen und Verhaltensweisen im Internet als Ressource und zugleich als formbar gemäss einer maximalen monetären Verwertbarkeit begreift - und dementsprechende Geschäftsmodelle etabliert, deren Fundament unter anderem in einer Verschiebung von Wissen, 
Autorität und Machtverhältnissen besteht (ebd., 180-182) -, ergibt sich ein ebenso kohärentes wie bedenkliches Bild. Die Akkumulation dieser drei Aspekte durch einige wenige globale Player wie Google, Amazon oder Facebook geht laut Zuboff einher mit noch nie dagewesenen Möglichkeiten der Steuerung menschlichen Verhalten bis hin zu ganzen Gesellschaften. In Anbetracht der umfassenden Gestaltungsansprüche grosser Internetplattformen werfen die in diesem Zusammenhang affirmativ erscheinenden Argumentationsmuster des $21^{\text {st }}$-Century-/4K-Skills-Diskurses aus pädagogischer Sicht Fragen auf. So lässt sich etwa fragen, inwiefern eine Orientierung entlang der vorgeschlagenen Bildungsinhalte Ansatzpunkte bietet, die hier nur kursorisch umrissenen Entwicklungen grundsätzlich infrage zu stellen. Die mit einem der 4Ks verbundene Forderung, kritisches Denken als Zielsetzung zu verfolgen, bietet zumindest keine Hinweise darauf, die von Zuboff dargestellten Entwicklungen generell im Sinne einer Systemkritik infrage zu stellen und Gegenentwürfe gegenüber der wirklichkeitskonstituierenden Kraft ökonomisch orientierter Internetplattformen anzuregen. Stattdessen wird in erster Linie die Identifikation von Potenzialen zur Optimierung der Selbstentfaltung gemäss der vorgegebenen bedingten Freiheit betont. Die Maxime ist hierbei die Einpassung in das präsentierte Kriterienschema des modernen Lernenden-Subjekts, in dessen Handeln - ähnlich wie vom unternehmerischen Selbst (Bröckling 2007) bekannt - individuelle und gesellschaftliche Ziele scheinbar widerspruchsfrei in einer ökonomisch geprägten Logik konvergieren.

Weiter fällt auf, dass der hier im Fokus stehende Diskurs auch einige Konzepte aufgreift, die bereits vor einigen Jahren im Zusammenhang mit einer zunehmenden Ökonomisierung des Bildungssystems diskutiert wurden. Zielsetzungen wie «Flexibility and Adaptability» (P21 2019, 6) oder «Initiative and Self-Direction» (ebd.) sind altbekannte «Plastikwörter» (Pongratz 2007), die schon im Zuge neoliberaler Umbauten im Bildungssystem zur Jahrtausendwende Einzug in den Diskurs hielten. Gleiches gilt auch für die Verheissungen eines erfüllten und erfolgreichen Lebens durch eine möglichst passgenaue Realisierung der proklamierten Zielsetzung, sprich: der ganzheitlichen Ausrichtung des Menschen auf die herangetragenen Zieldimensionen. «Der radikale Umbruch erzeugt Druck und Angst», heisst es bei Pongratz (2019, 346), und zugleich: «[V]ersüßt werden die Verunsicherungen jedoch mit dem Versprechen, am Ende winke ein Bildungseldorado, das die Bildungsbedürfnisse von mehr Menschen als je zuvor zufrieden stellen können» (ebd., 346f.). Diese in Bezug auf die Bildungsreformen zu Beginn der 2000er Jahre gerichtete Analyse scheint mehr denn je auch für den hier betrachteten Zusammenhang zu gelten. ${ }^{21}$

21 An dieser Stelle sei auf ein weiteres Detail hingewiesen, das hier aus Platzgründen nicht weiter ausgeführt werden kann: Interessant erscheinen auch die nicht vollzogenen Anschlüsse an pädagogische Diskurslinien. Keine Rolle spielen etwa Open Educational Ressources (OER), ganz im Unterschied zur Entwicklung gegenwärtiger medienpädagogischer Debatten, in denen diese zunehmend als wichtiger Bestandteil einer zeitgemässen Öffnung des Bildungssystems gesehen werden. Ebenso wenig werden Fragen von Diversität oder postkolonialer Ungleichheiten im 4K-/21 $1^{\text {st }}$-Century-Skills-Diskurs aufgegriffen. 


\section{Diskussion und Fazit}

Der 21 $1^{\text {st }}$-Century-/4K-Skills-Diskurs «lebt von der Behauptung, vergleichsweise griffige und einfache Lösungen für eine komplexe Problemlage zu bieten. Die Klarheit und scheinbare Eindeutigkeit, mit der Lösungsvorschläge dargeboten werden, führt aber aus erziehungswissenschaftlicher Perspektive instinktiv zur Skepsis. Die hier geübte Kritik soll - das ist an dieser Stelle zu betonen - nicht als Beharren auf traditionellen Formen und Formaten von Bildung missverstanden werden. Die Reformbedürftigkeit des Bildungssystems ist, nicht zuletzt aufgrund des gesellschaftlichen Wandels, den

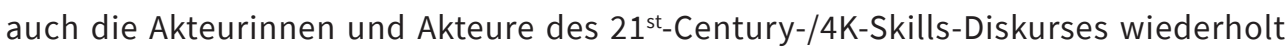
betonen, kein realitätsfernes Anliegen und auch aus erziehungswissenschaftlicher Perspektive durchaus begründbar.

Die hier dargestellte Entwicklung lässt sich - kurz gesagt - als logische Konsequenz einer weiter voranschreitenden Privatisierung und Ökonomisierung des Bildungswesens beschreiben, die in globalem Massstab vorangetrieben wird. Es ist aus dieser Perspektive sicher wenig überraschend, dass der Diskurs - wie eingangs erwähnt - nicht nur seinen Ursprung im US-amerikanischen Raum hat, sondern hier offenbar auch seine grösste Wirkung entfaltet und andersherum etwa im deutschen

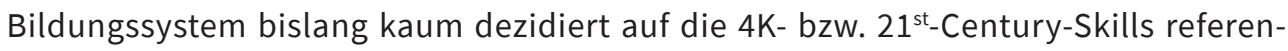
ziert wird. ${ }^{22}$ Wenngleich diese Differenzen nicht unbedacht bleiben dürfen, scheint der global vernetzte Kontext und die Historie des Bildungsreformdiskurses wichtig, in deren Licht die oben dargelegten Entwicklungen zu sehen sind. Die Diagnose einer Neudefinition der Rolle von Schülerinnen und Schülern als 'Selbstmanager:innen des Wissens), die Pongratz $(2004,254)$ mit Blick auf Bildungsreformen der 2000er Jahre skizziert, kann mit Blick auf die im $4 \mathrm{~K}-/ 21^{\text {st }}$-Century-Skills-Diskurs entfalteten Positionen durchaus in einer aktualisierten Form - versehen mit neuen Schlagworten - wiedergefunden werden. Als erstaunlich aktuell in dem hier betrachteten Zusammenhang erweisen sich auch die «Maximen der 〈Viabilität) und ‘Anschlussfähigkeit»» (ebd., 255) als Indikatoren von Lernerfolg. Die Rede von Lernenden als «Versatilisten» (Fadel, Bialik, und Trilling 2017, 3) fügt sich nicht nur weitgehend nahtlos in diese Metaphorik ein, sondern bringt auch eine sehr ähnliche Zielvorstellung zum Ausdruck, deren Kern sich als ein Set von flexibel zum Einsatz kommenden Selbstkontroll- und -reflexionstechnologien auszeichnet. Insofern scheint es nicht abwegig anzunehmen, dass Transformationsbemühungen im Sinne der $21^{\text {st }}$-Century-/4K-Skills auch im europäischen Raum auf fruchtbaren Boden fallen, den vergangene Bildungsreformen bereitet haben, die insbesondere im Hochschulsektor bereits zu erheblichen

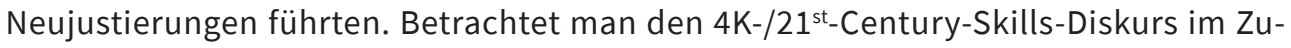
sammenhang einer globalen Geopolitik des Wissens (Zelinka i. E.), werden - etwa

22 Dies soll jedoch nicht pauschal als positive Einschätzung missverstanden werden, da dies zu einem Gutteil an der generellen Reformträgheit des deutschen Bildungssystems liegen könnte, die selbst wiederum nicht unproblematisch ist. 
in Form der Definitionsmacht über Formen globaler Wissensproduktion durch eine exklusive Gruppe von Akteurinnen und Akteuren - die hier wirksamen hegemonialen Positionen deutlich. Die Ansprüche der Reformen, einen umfassenden und tiefgreifenden Veränderungsprozess auslösen zu wollen, der eine Unterordnung unter die dargebotene Zielvorgabe erfordert, verdeutlicht diese machtvolle Stellung. ${ }^{23}$ Auch wenn Reformbewegungen in dieser Hinsicht im deutschsprachigen Raum bislang nur wenig fortgeschritten sind, zeigen die oben unternommenen Erkundungen doch die Brisanz und Dringlichkeit, sich aus (medien-)pädagogischer Perspektive mit diesen Diskursen zu befassen und eine eigene Position zu entwickeln.

\section{Literatur}

Alkemeyer, Thomas, Ulrich Bröckling, und Tobias Peter, Hrsg. 2018. Jenseits der Person. Zur Subjektivierung von Kollektiven. Bielefeld: transcript. https://doi.org/10.14361/9783839438428002.

Anhorn, Roland, Frank Bettinger, und Johannes Stehr, Hrsg. 2007. Foucaults Machtanalytik und soziale Arbeit: eine kritische Einführung und Bestandsaufnahme. Perspektiven kritischer sozialer Arbeit. Wiesbaden: Springer VS. https://doi.org/10.1007/978-3-531-90710-9.

Aufenanger, Stefan. 2017. «21st Century Skills». Computer + Unterricht 28, 4-7.

Battelle or Kids. 2020. «Annual Report 2020». http://static.battelleforkids.org/Documents/BFK/ BFK-Annual-Report-2020.pdf.

Bellanca, James, und Ron Brandt, Hrsg. 2010. 21st Century Skills: Rethinking How Students Learn. Bloomington: Solution Tree.

Bröckling, Ulrich. 2007. Das unternehmerische Selbst. Soziologie einer Subjektivierungsform. Frankfurt a.M.: Suhrkamp.

Bröckling, Ulrich. 2017. Gute Hirten führen sanft. Über Menschenregierungskünste. Berlin: Suhrkamp.

Bröckling, Ulrich, Susanne Krasmann, und Thomas Lemke, Hrsg. 2012. Gouvernementalität der Gegenwart. Studien zur Ökonomisierung des Sozialen, 6. Aufl., Frankfurt a. M.: Suhrkamp.

Bühler, Patrick, Edgar Forster, Sascha Neumann, Sabrina Schröder, und Daniel Wrana, Hrsg. 2015. Normalisierungen. Halle-Wittenberg: Martin-Luther-Universität.

Cheney-Lippold, John. 2019. We are Data. Algorithms an the Making of our Digital Selves. New York: New York University Press. https://doi.org/10.1177/1461444818806495.

Dander, Valentin. 2018. «Medienpädagogik Im Lichte | Im Schatten Digitaler Daten. Manteltext». MedienPädagogik: Zeitschrift für Theorie Und Praxis Der Medienbildung, 1-134. https://doi.org/10.21240/mpaed/diss.vd.01.X.

23 Verdeutlichen lässt sich dies exemplarisch an folgendem Zitat: «For enduring transformation, all aspects of the system - including the strategic plan - must be aligned and anchored to the vision for 21st century learning, the Portrait of a Graduate» (Battelle for Kids 2020, 10). 
Dzierzbicka, Agnieszka. 2006. Vereinbaren statt anordnen. Neoliberale Gouvernementalität macht Schule. Wien: Löcker.

Esposito, Elena. 2007. Die Fiktion der wahrscheinlichen Realität. Frankfurt a. M.: Suhrkamp.

Fadel, Charles. 2015. «Redesigning the Curriculum for a 21st Century Education. The CCR Foundational White Paper». https://curriculumredesign.org/wp-content/uploads/CCRFoundationalPaper-Updated-Jan2016.pdf.

Fadel, Charles, Maya Bialik, und Bernie Trilling. 2015. Die Vier Dimensionen Der Bildung. Was Schülerinnen Und Schüler Im 21. Jahrhundert Lernen Müssen [Four-Dimensional Education: The Competencies Learners Need to Succeed]. Übersetzt von Jöran Muuß-Merholz. Boston, MA: Center for Curriculum Redesign.

Fegter, Susann, Fabian Kessl, Antje Langer, Marion Ott, Daniela Rothe, und Daniel Wrana. 2015. «Erziehungswissenschaftliche Diskursforschung. Theorien, Methodologien, Gegenstandskonstruktionen». In Erziehungswissenschaftliche Diskursforschung. Empirische Analysen zu Bildungs- und Erziehungsverhältnissen, herausgegeben von Susann Fegter, Fabian Kessl, Antje Langer, Marion Ott, Daniela Rothe, und Daniel Wrana, 9-55. Wiesbaden: Springer VS. https://doi.org/10.1007/978-3-531-18738-9_1.

Forneck, Hermann J., und Daniel Wrana. 2005. «Ein parzelliertes Feld. Eine Einführung in die Erwachsenenbildung». Bielefeld: Bertelsmann. https://doi.org/10.25656/01:19897.

Foucault, Michel. 2012. «Die «Gouvernementalität». In Gouvernementalität der Gegenwart. Studien zur Ökonomisierung des Sozialen, herausgegeben von Ulrich Bröckling, Susanne Krasmann, und Thomas Lemke, 6.Aufl., 41-67. Frankfurt a. M.: Suhrkamp.

Foucault, Michel 2019. «Geschichte der Gouvernementalität I. Sicherheit, Territorium, Bevölkerung. Vorlesung am Collège de France 1977-1978». Frankfurt a. M.: Suhrkamp.

Global Partnership for Education. 2020. «21st-Century Skills: What potential role for the Global Partnership for Education? A Landscape Review». https://www.globalpartnership.org/sites/ default/files/document/file/2020-01-GPE-21-century-skills-report.pdf.

Höhne, Thomas. 2015. «Ökonomisierung, Interdiskurs, Bildung. Eine diskursanalytische Perspektive auf die Normalisierung ökonomischer Logiken im Bildungsbereich» In Normalisierungen, herausgegeben von Patrick Bühler, Edgar Forster, Sascha Neumann, Sabrina Schröder und Daniel Wrana, 57-75. Halle-Wittenberg: Martin-Luther-Universität.

Kauppinen, Kati. 2012. «Subjects of Risk. Neoliberale Gouvernementalität in einer gegenwärtigen Frauenzeitschrift». In Mediendiskursanalyse. Diskurse - Dispositive - Medien - Macht, herausgegeben von Philipp Dreesen, Łukasz Kumięga, und Constanze Spieß, 189-206. Wiesbaden: Springer VS. https://doi.org/10.1007/978-3-531-93148-7_8.

Kultusministerkonferenz [KMK]. 2012. «Medienbildung in der Schule. Beschluss der Kultusministerkonferenz». https://www.kmk.org/fileadmin/veroeffentlichungen_beschluesse/2012/2012_03_08_Medienbildung.pdf.

Kultusministerkonferenz [KMK]. 2017. «Strategie der Kultusministerkonferenz,Bildung in der digitalen Welt'». https://www.kmk.org/fileadmin/Dateien/pdf/PresseUndAktuelles/2018/ Digitalstrategie_2017_mit_Weiterbildung.pdf. 
Kreß, Jennifer. 2018. «Digitale Bildung aus der Perspektive der USA». Medien + Erziehung 62, 42-44.

Lang, Katarina. 2013. «Thank you for not smoking» - Zur Gouvernementalität des Rauchens». Medienwelten - Zeitschrift für Medienpädagogik (1): 1-135. https://doi.org/10.13141/ zfm.2013-1.46.

Lemke, Thomas, Susanne Krasmann, und Ulrich Bröckling. 2012. "Gouvernementalität, Neoliberalismus und Selbsttechnologien. Eine Einleitung». In Gouvernementalität der Gegenwart. Studien zur Ökonomisierung des Sozialen, herausgegeben von Ulrich Bröckling, Susanne Krasmann, und Thomas Lemke, 6. Aufl., 7-40. Frankfurt a. M.: Suhrkamp.

Marttila, Tomas. 2014. «Die wissensbasierte Regierung der Bildung - Die Genese einer transnationalen Gouvernementalität in England und Schweden» Berliner Journal für Soziologie 24 (2): 257-287. https://doi.org/10.1007/s11609-014-0247-6.

Maurer, Susanne, und Susanne Weber. 2006. «Die Kunst, nicht dermaßen regiert zu werden. Gouvernementalität als Perspektive für die Erziehungswissenschaft». In Gouvernementalität und Erziehungswissenschaft. Wissen - Macht - Transformation, herausgegeben von Susanne Weber und Susanne Maurer, 9-36. Wiesbaden: Springer VS. https://doi. org/10.1007/978-3-351-90194-7_1.

Mehta, Rohit, Edwin Creely, und Danah Henriksen. 2020. «A Profitable Education: Countering Neoliberalism in 21st Century Skills Discourses.» In Handbook of Research on Literacy and Digital Technology Integration in Teacher Education, herausgegeben von Jared Keengwe und Grace Onchwari, 359-381. Hershey, PA: IGI Global. https://doi.org/10.4018/978-17998-1461-0.ch020.

Münte-Goussar, Stephan. 2011. «Ambivalente Selbst-Techniken: Portfolio, Ökonomisierung, Selbstbestimmung». In Kontrolle und Selbstkontrolle. Zur Ambivalenz von E-Portfolios in Bildungsprozesse, herausgegeben von Torsten Meyer, Kerstin Mayrberger, Stephan Münte-Goussar und Christina Schwalbe, 225-249. Wiesbaden: Springer VS. https://doi. org/10.1007/978-3-351-92722-0_20.

Neurath, Wolfgang. 2017. «Von den Dispositiven der Macht und ihrer sozialen Morphologie: Zur Aktualität der Machtanalysen Michel Foucaults». Medienimpulse 54 (4): 1-30. https://doi. org/10.21243/medienimpulse.2016.4.1028.

Niesyto, Horst. 2017. «Medienpädagogik und digitaler Kapitalismus. Für die Stärkung einer gesellschafts- und medienkritischen Perspektive». MedienPädagogik 27: 1-29. https://doi. org/10.21240/mpaed/27/2017.01.13.X.

Ntemiris, Nektarios. 2011. "Gouvernementalität und Kindheit. Transformationen generationaler Ordnung in Diskursen und in der Praxis». Wiesbaden: Springer VS. https://doi. org/10.1007/978-3-531-92827-2.

P21. 2019. «Framework for 21st Century Learning Definitions». http://static.battelleforkids.org/ documents/p21/P21_Framework_DefinitionsBFK.pdf.

Pongratz, Ludwig. 1988. «Michel Foucault: Seine Bedeutung für die historische Bildungsforschung.» Information zur erziehungs- und bildungshistorischen Forschung (IZEBF) 32, 155168. 
Pongratz, Ludwig. 1990. «Schule als Dispositiv der Macht. Pädagogische Reflexionen im Anschluss an Michel Foucault.» Vierteljahrsschrift für wissenschaftliche Pädagogik 66 (3): 289-308.

Pongratz, Ludwig. 2004. «Freiwillige Selbstkontrolle. Schule zwischen Disziplinar- und Kontrollgesellschaft». In Michel Foucault: pädagogische Lektüren, herausgegeben von Norbert Ricken und Markus Rieger-Ladich, 243-259. Wiesbaden: Springer VS. https://doi. org/10.1007/978-3-322-85159-8_13.

Pongratz, Ludwig 2007. «Plastikwörter». Notizen zur Bildungsreform, Engagement (3): 161170.

Pongratz, Ludwig A. 2009. «Pädagogische Gouvernementalität Und Neoliberale Regierungsweise - Gewinn und Grenze pädagogischer Gouvernementalitäts-Studien». Jahrbuch Für Pädagogik (1): 187-99. https://doi.org/10.3726/92148_187.

Pongratz, Ludwig A. 2010. «Freiheit und Kontrollgesellschaft Gouvernementale Strategien der Bildungsreform (2008)». In Sammlung. Fundstücke aus 30 Hochschuljahren, herausgegeben von Ludwig A. Pongratz. Darmstadt: tuprints. http://nbn-resolving.de/urn:nbn:de:tudatuprints-24395.

Raithelhuber, Eberhard. 2006. «Netzwerk der Politikgestaltung im Sozial- und Bildungssektor ein Blick durch die Brille der Gouvernementalität». In Gouvernementalität und Erziehungswissenschaft. Wissen - Macht - Transformation, herausgegeben von Susanne Weber und Susanne Maurer, 163-179. Wiesbaden: Springer VS. https://doi.org/10.1007/978-3-53190194-7_9.

Ricken, Norbert, und Markus Rieger-Ladich, Hrsg. 2004. Michel Foucault: pädagogische Lektüren. Wiesbaden: Springer VS. https://doi.org/10.1007/978-3-322-85159-8.

Rieger, Stefan 2018. Freiwillige Fremdkontrolle. Paradoxien der Gouvernementalität. In Unterwachen und Schlafen. Anthropophile Medien nach dem Interface, herausgegeben von Michael Andreas, Dawid Kasprowicz, Stefan Rieger, 49-75. Lüneburg: meson press, https://doi. org/10.25969/mediarep/1571.

Rotherham, Andrew. J., und Daniel Willingham. 2009. «21st Century Skills the Challenges ahead». Educational Leadership 67 (1): 16-21. https://www.ascd.org/el/articles/21st-century-skills-the-challenges-ahead.

Schröder, Sabrina, und Daniel Wrana. 2015. «Normalisierungen- eine Einleitung». In Normalisierungen, herausgegeben von Patrick Bühler, Edgar Forster, Sascha Neumann, Sabrina Schröder und Daniel Wrana, 9-34. Halle-Wittenberg: Martin-Luther-Universität.

Seyfert, Robert, und Jonathan Roberge, Hrsg. 2017. "Algorithmuskulturen. Über die rechnerische Konstruktion der Wirklichkeit». Bielefeld: transcript. https://doi. org/10.14361/9783839438008_001.

Simons, Maarten. 2004. «Lernen, Leben und Investieren: Anmerkungen zur Biopolitik». In Michel Foucault: pädagogische Lektüren, herausgegeben von Norbert Ricken und Markus Rieger-Ladich, 165-185. Wiesbaden: Springer VS. https://doi.org/10.1007/978-3-322-851598_9. 
Skrandies, Timo. 2014. «Mediale Gouvernementalität» In Wissen und Leben - Wissen für das Leben, herausgegeben von Vittoria Borsò, 281-304. Bielefeld: transcript. https://doi. org/10.14361/transcript.9783839421604.281.

Stauff, Markus. 2005. «Das neue Fernsehen: Machtanalyse, Gouvernementalität und digitale Medien». Münster: Lit.

Stauffer, Bri (2020). «What Are 21st Century Skills?» https://www.aeseducation.com/blog/whatare-21st-century-skills.

Sterel, Saskia, Manfred Pfiffner, und Claudio Caduff. 2018. «Ausbilden nach 4K. Ein Bildungsschritt in die Zukunft». Bern: hep.

Zelinka, Jozef. (i. E.). "Subjects and Subjectivities of the (new) Geopolitics of Knowledge». In Geopolitical Transformations in Higher Education, herausgegeben von Marcelo Parreira do Amaral und Christiane Thompson, Wiesbaden: Springer VS.

Zuboff, Shoshana. 2019. «The Age of Surveillance Capitalism: The Fight for the Future at the New Frontier of Power». London: Profile Books. 